\title{
Bioequivalence Guidelines Requirements for Orally Administered Generics (IR Products) in Gulf Cooperation Council Countries, European Union and United States of America
}

\author{
Elambharathy Elangovan*
}

Department of Bioequivalence, Research Ë Development, Jamjoom Pharmaceuticals, P.O. Box \# 6267, Jeddah-21442, Kingdom of Saudi Arabia

\begin{abstract}
The importance of the bioequivalence requirements is increasing in the GCC (Gulf Cooperation Council) region pertaining towards the growth of generic drug market. GCC bioequivalence guidelines describes the requirements for bioequivalence study as per this region regulatory requirements. This review article mainly focuses on bioequivalence study requirements in the Gulf Cooperation Council countries, European union countries and the United States of America that needs to be fulfilled in order to successfully submit a generic application. This comparison includes the bioequivalence approaches such as study design, sample size, study condition, pharmacokinetic parameters, statistical analysis, narrow therapeutic index drugs, highly variable drug products and BCS based bio waiver requirements. The rationale for this article is to give prompt overview on the bioequivalence requirements in these region and in comparison with well-established regulatory such as USFDA and EMA. In addition, it also highlights the possibilities for harmonization.

Keywords: Bioavailability; Bioequivalence; Biowaiver; Pharmacokinetics

Abbreviation: GCC: Gulf Cooperation Council; EMA: European Medicines Agency; USFDA: U.S. Food \& Drug Administration; $\mathrm{C}_{\max }$ : Maximum Plasma Concentration; AUC(0-t): Area Under the Plasma Concentration Curve from Administration to Last Observed Concentration at Time t; AUC $(0-\infty)$ : Area Under the Plasma Concentration

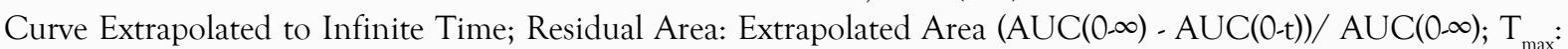
Time Until $\mathrm{C}_{\max }$ is Reached; $\lambda z$ : Terminal Rate Constant; t1/2: Plasma Concentration Half-life; AUC(0-72 h): Area Under the Plasma Concentration Curve from Administration to $72 \mathrm{~h}$; AUC $(0-\tau)$ : AUC During a Dosage Interval at Steady State; $\mathrm{C}_{\text {max }, \mathrm{ss}}$ : Maximum Plasma Concentration at Steady State; $\mathrm{t}_{\max , \mathrm{ss}}$ : Time Until $\mathrm{C}_{\max , \mathrm{ss}}$ is Reached; $\mathrm{C}_{\min }$ : Concentration at the End of a Dosing Interval; Cav: Average Concentration During a Dosing Interval; Ae(0-t): Cumulative Urinary Excretion of Unchanged Drug from Administration until Time $t ; \mathrm{R}_{\max }$ : Maximal Rate of Urinary Excretion; NTIDs: Narrow Therapeutic Index Drugs; OGD: Office of Generic Drugs; PK: Pharmacokinetics; BA: Bioavailability; BE: Bioequivalence; Stat: Statistics; RLD: Reference Listed Drug; CI: Confidence Interval; HVDP: Highly Variable Drug Products; USP: United States Pharmacopeia; BCS: Biopharmaceutics Classification System; IR: Imeediate Release; FIP: International Pharmaceutical Federation; CV: Coefficient Variation; BMI: Body Mass Index.
\end{abstract}

\section{INTRODUCTION}

This review article mainly focuses on the bioequivalence study requirements in the Gulf Cooperation Council countries, European union countries and the United States of America. The comparison between the GCC, USFDA and EMA regulatory requirements will be useful for fraternity in the pharmaceutical industry, those who are in the generic product development to understand the local regulatory requirements in comparison with well-established regulatory such as USFDA and EMA. Since, GCC guidelines adherence to the EMA guidelines approaches to establish bioequivalence is almost similar and however in some aspects difference are there between both guidance. This comparison

Correspondence to: Elambharathy Elangovan, Department of Bioequivalence, Research \& Development, Jamjoom Pharmaceuticals, P.O. Box \# 6267, Jeddah-21442, Kingdom of Saudi Arabia, Tel: +966 558652821; E-mail: elampharmacologist@gmail.com

Received: November 27, 2018, Accepted: February 08, 2019, Published: February 15, 2019

Citation: Elangovan E (2019) Bioequivalence Guidelines Requirements for Orally Administered Generics (IR Products) in Gulf Cooperation Council Countries, European Union and United States of America. J Bioequiv Availab 11:387. doi: 10.35248/0975-0851.19.11.387

Copyright: ( 2019 Elangovan E. This is an open-access article distributed under the terms of the Creative Commons Attribution License, which permits unrestricted use, distribution, and reproduction in any medium, provided the original author and source are credited. 
will give clear aspects in understanding the similar and difference between these regulatory requirements for conducting the studies. It also helpful to the academic professionals, to understand the regulatory requirements for conduct the bioavailability and bioequivalence studies.

\section{GENERAL APPROACHES TO ESTABLISH BIOEQUIVALENCE}

\section{Design of the study}

Two-way crossover study is the unique standard study design recommended by GCC, EMA \& USFDA. For long half-life drugs EMA \& GCC recommends parallel design. USFDA recommends parallel design, if they have faced any problem with crossover study. For highly variable drugs EMA \& GCC recommends replicate design. USFDA recommendation is reference scaled average bioequivalence study.

\section{Sample size}

USFDA recommends pilot study, before proceeding with a full bioequivalence study. EMA \& GCC not specific in the requirement of pilot study, at the same time EMA recommends 12 subjects for bioequivalence study. Hence, it confirms EMA recommends minimum 12 subjects required for either pilot or pivotal study. GCC recommends 24 subjects for pivotal bioequivalence study. USFDA not specific in minimum subject requirement, but in the earlier guidance mentioned about sample size requirements (e.g., 12).

\section{Selection of reference product}

Reference product should be marketed in EU as per the EMA recommendations. RLD (Reference Listed Drug) is the reference drug for USFDA submissions. GCC recommends the reference product must be the original brand-name (i.e. manufactured in the country of origin of the original brand name); if unable to get the product in that market then the brand-name regarding the same company but different country of origin is used, marketed in GCC region, ICH region, or in any stringent regulatory authority. If unable to get the original brand-name product in the market or not manufactured, then the local country market leader may be used as a reference product. However, sometimes it's difficult to get the details of innovator and its country of origin. It means whether it's first launched in the U.S. or Europe or some other places. Hence, in the GCC guidelines, acceptancy of both U.S. and Europe region available reference product in consideration of well-established market, would be helpful to do the selection of the reference product.

\section{Study conditions}

As per the EMA \& GCC, fasting time for the subjects is minimum at least 8 hours before dosing of the drug products. USFDA recommends minimum 10 hours fasting before dosing. Food instructions are similar to these for fed studies to all these three regulatory. For fed studies, generally high fat (approximately half of the total caloric content of the meal), high-calorie (approximately 800 to 1000 calories) meal is recommended as a breakfast. Accordingly, the breakup for 1000 calories are 150 calories from protein, 250 calories from carbohydrate and 500-600 calories from fat.

\section{Pharmacokinetic (PK) parameters}

Pharmacokinetic parameters such as AUC (0-t), AUC $(0-\infty)$, residual area, $\mathrm{C}_{\max }, \mathrm{t}_{\max }, \square z$, and $\mathrm{t} 1 / 2$ need to be evaluated in the bioequivalence study. The requirement of steady state studies submission in EMA \& GCC are AUC (0- $), \mathrm{C}_{\text {max,ss }}$, and $t_{\text {max,ss }}$. USFDA recommends $\mathrm{C}_{\text {min }}$ (concentration at the end of a dosing interval), Cav (average concentration during a dosing interval), degree of fluctuation $\left[\left(\mathrm{C}_{\max }-\mathrm{C}_{\min }\right) / \mathrm{Cav}\right]$, and swing $\left[\left(\mathrm{C}_{\max }-\mathrm{C}_{\min }\right) /\right.$ $\mathrm{C}_{\text {min }}$ ] if steady-state studies are employed. Ae(0-t) and, if applicable, Rmax need to be evaluated when using urinary data for EMA \& GCC. Product specific guidance need to be followed for USFDA submission.

\section{Acceptance criteria for PK parameters}

The acceptance criteria is the point estimate between test and reference products for $90 \%$ CI values within the acceptance range of $80.00-125.00 \%$. For NTI drugs, GCC and EMA recommends the acceptance range for AUC \& $\mathrm{C}_{\text {max }}$ should be tightened to 90.00 $111.11 \%$. As per USFDA, if it is mentioned in the product specific guidance accordingly it will be applicable to the products. USFDA guidance describes, the general acceptance range of $80 \%$ to $125 \%$ for non-NTI products will not be changed for the bioavailability measures (AUC and $\mathrm{C}_{\max }$ ) of NTI drugs. For Highly variable drugs, GCC and EMA guidance states that $\mathrm{C}_{\text {max }}$ can be extended (i.e. 75 $133 \%$ ). USFDA suggests, scaled-average BE analysis approach for highly variable drugs.

\section{Baseline correction}

GCC, EMA and USFDA highlights that authorities to check the baseline endogenous levels in plasma and subtract these plasma concentrations from the total endogenous amount measured from every subject after the post dose. In GCC \& EMA subtracting individual endogenous pre-dose AUC, is recommended.

\section{Measurement of metabolite}

As per USFDA, the analytes to be quantified in biological fluids collected in BA and BE studies are either the active drug ingredient or parent drug or, if it's required then active metabolites. GCC and EMA recommend, bioequivalence need to be concluded upon determined amount of the drug. It's because of that $\mathrm{C}_{\max }$ of a drug is generally high sensitive to determine the differences between drugs in absorption. In certain conditions, particularly for some pro-drugs demonstrating bioequivalence only for the main active metabolite is acceptable.

\section{Drug proportion difference}

USFDA, EMA and GCC recommends, 5\% difference is acceptable for the assay content between the test and reference product and it shouldn't be $>5 \%$.

\section{Retention period for drugs and documents and quantity of drugs}

As per USFDA, remaining samples of both the test and the reference drugs to be retained at the study site for a period of 5 years. This is because, OGD to do the release tests for five times. The maximum limit of 300 units each for the test and reference drugs can be 
considered enough to do the release test in consideration of the oral solid dosage forms. No specific recommendations from the GCC and EMA for retention period and quantity of drugs.

Guidance for Industry, Handling and Retention of BA and BE Testing Samples, U.S. Department of Health and Human Services, Food and Drug Administration, Center for Drug Evaluation and Research (CDER) [1].

European Medicines Agency, London, 20 January 2010, (Doc. Ref.: CPMP/EWP/QWP/1401/98 Rev. 1/ Corr), Committee for Medicinal Products for Human Use, Guideline on the investigation of bioequivalence [2].

Executive Board of the Health Ministers' Council for GCC States, the GCC Guidelines for Bioequivalence, Version 2.4, MAR 2016 [3].

\section{Enantiomers}

EMA and GCC guidelines states, achiral bioanalytical methods are usually acceptable. The below mentioned conditions to be met when the individual enantiomers to should be measured:

1) The enantiomers exhibit different pharmacokinetics

2) The enantiomers exhibit pronounced difference in pharmacodynamics

3) The exposure (AUC) ratio of enantiomers is modified by a difference in the rate of absorption.

It is enough to demonstrate the bioequivalence for the active enantiomer, if one is pharmacologically active and another one is inactive or low active. USFDA requirements are, for BA studies, important one is quantification of individual enantiomers. For BE studies, important one is quantification of the racemate using an achiral assay. The below mentioned conditions to be met when the individual enantiomers to should be measured:

(1) The enantiomers exhibit different pharmacodynamic characteristics

(2) The enantiomers exhibit different pharmacokinetic characteristics

(3) Primary efficacy and safety activity resides with the minor enantiomer

(4) Nonlinear absorption is present for at least one of the enantiomers. In such cases, we recommend that BE factors be applied to the enantiomers separately. In comparison with the EMA requirements, USFDA recommends additionally to ensure the efficacy and safety activity for the minor enantiomer.

\section{Endogenous substances}

Endogenous compounds are drugs that body already having either because it naturally present in the body and getting from the normal diet. Endogenous substances are identical to drug and hence its quiet difficult to find the quantification of drug released from the dosage and absorbed by the body.

EMA and GCC guide us, baseline correction need to be performed to understand the additional concentrations due to the treatment during the endogenous substance analysis.
However, before study proceedings the correcting baseline to be mentioned and justified in the protocol. Baseline correction usually follows the normal subtraction, it means individual mean endogenous pre-dose concentrations to be subtracted or endogenous pre-dose AUC is recommended for subtraction.

USFDA recommends specifically for periods. If a baseline corrected values in negative, the value should be set equal to 0 before the baseline-corrected AUC calculation. Final PK \& stat analysis to be conducted on the both corrected and uncorrected data. However, conclusion of bioequivalence based on the baseline- corrected data.

\section{Investigation strength}

GCC and EMA recommends, either one or two strength is sufficient to conclude the bioequivalence, it depends on the proportionality of the different strengths and other product related issues described below. Drug linearity is important factor to finalize the strength to be selected for BE. If the drug doesn't obeys the linearity that means non-linear pharmacokinetics (i.e. if we increase the dose the AUC increase is not proportional), then it would be the difference of different strengths in the sensitivity to identify the differences between formulations. Linearity is described as if the dose adjusted mean AUCs is not $>25 \%$ difference in comparison with the BE study conducted drug strength and for the biowaiver strengths. Generally, higher strength would be selected for the bioequivalence study. If the drug obeys the linearity in PK and also high solubility and then selection of a lower strength is acceptable instead of higher strength. However, in consideration of safety/tolerability issues lower strength also can be selected with justification. USFDA accepts the lower strength in consideration of safety, as per the below mentioned conditions acceptancy: i) Linear elimination kinetics has been documented over the therapeutic dose range. ii) The higher strengths of the test and RLD products are proportionally similar to their corresponding lower strength. iii) Comparative dissolution testing on the higher strength of the test and RLD products has been submitted and found to be acceptable. In general, waiver can be accepted based on (i) acceptable BE study on the designated strength, (ii) acceptable in vitro dissolution testing of all the strengths, and (iii) proportional similarity of the formulations across all strengths.

\section{Evaluation}

The assay content difference, in general should not be adjusted in the PK parameters of the test and reference batch as per the EMA and GCC bioequivalence guidelines recommendations. The same can be acceptable if the assay content difference is less than $5 \%$ for test product in comparison with reference and can't be found content correction. In protocol, it should be pre-specified if content correction is to be done and also justified by inclusion of the results from the assay.

Subject accountability: Statistical analysis need to be done in the all dosed subjects. However, in a crossover study if the evaluable data is not available then both test and reference products should not be included.

Subject exclusion: Exclusion of any of the subject to be decided before initiation of the bioanalysis and the reason for exclusion need to be mentioned in the protocol. Excluding data is not usually acceptable in consideration of statistical analysis or due to PK 
reasons, because it's not possible to differentiate the formulation effects from other effects influencing the pharmacokinetics.

However, some exceptions are as mentioned below;

1) Any subject is having lack of measurable concentration or very low plasma concentrations for reference medicinal product. If the subject AUC is less than $5 \%$ of reference medicinal product geometric mean AUC then it is considered as very low plasma concentrations. This reason is usually accepted in exceptional cases for exclusion of data; however it may question the validity of the study.

2) Any subjects having non-zero baseline concentrations of more than $5 \% \mathrm{C}_{\max }$, that data need to be excluded from bioequivalence calculation. It is applicable for immediate release formulations, due to the reason of subject non-compliance and an insufficient wash-out period, respectively, and poor internal dosing and study procedures such as mouth check of subjects after dosing to ensure the subjects have swallowed the study medication and study design with adequate wash out. Even though the subjects excluded from the statistical analysis, the samples to be assayed and the results listed. Moreover, AUC (0-t) should cover at least $80 \%$ of $\mathrm{AUC}(0-\infty)$. If any of the subjects, covers AUC (0-t) less than $80 \%$ of AUC $(0-\infty)$ should not be excluded from the statistical analysis. However, it's not acceptable if less than $80 \%$ in more than $20 \%$ of the observations. If it is more than $20 \%$ then the validity of the study is questionable. This procedure is not applicable for the truncated study design (72 h).

PK parameters: $C_{\max }, A U C(0-t)$, or, if it is required AUC(0-72h) are the parameters need to be evaluated and the $90 \% \mathrm{CI}$ for the ratio of the test and reference products should be within 80.00 $125.00 \%$ for these parameters.

Statistical analysis: In general, all the data need to be transferred to logarithmic transformation and follow up with it should analysed by using ANOVA for all the pharmacokinetic parameters. The $90 \%$ CI for the T/R of both products would be considered for the bioequiovalence assessment. This procedure is equivalent to two one-sided tests with the null hypothesis of bioinequivalence at the $5 \%$ significance level.

Carry-over effects: During statistical evaluation, subjects will be excluded in that period if the pre-dose concentration of $\mathrm{C}_{\max }$ is more than $5 \%$. In this type of case, the subject will be removed from the analysis if it as a two-way crossover study. However, due to these exclusions if the sample size is reduced to less than 12 subjects, then the study will not be acceptable. This statistical exclusion procedure is not applicable for the endogenous drugs.

Study design with two-stage: Study design with two-stage is acceptable and it should be mentioned in the protocol. In case the bioequivalence has not established in the first study then an additional or second stage study can be conducted and the results from two stages combined in the final analysis results.

\section{Narrow therapeutic index drugs}

Narrow therapeutic index drugs (NTIDs) required range for pharmacokinetic parameters such as $\mathrm{AUC}$ and $\mathrm{C}_{\text {max }}$ to be narrowed to $90.00-111.11 \%$, in consideration of safeness and effectiveness of the drug.

\section{Requirements for highly variable drug products (HVDP)}

In USFDA, EMA \& GCC, if the drug products show more than $30 \%$ intra subject variability and it would be considered as Highly Variable Drug Products (HVDP). Replicate study design approach is recommended by USFDA for HVDP products. In these replicate study design, both reference-scaled and complete replicate designs are acceptable as per the product requirements. In this referencescaled approach intra subject variability of the reference product is getting average and the range is 0.8 to 1.25 on the geometric mean ratio. In EMA and GCC, the same replicate cross-over design study can be carried out. Moreover, widened $\mathrm{C}_{\text {max }}$ difference is acceptable by proper clinical justification with an increased passing limits. In addition, $\mathrm{C}_{\max }$ can be increased to $69.84-143.19 \%$ and $75-133 \%$ respectively for EMA and GCC. Moreover, in EMA based on the different variabilities the different acceptance limits as mentioned in the below table. The procedure of widening the $\mathrm{C}_{\max }$ passing limits is only applicable for the study if we have applied the replicate design in which the ISCV should be $>30 \%$ in the $\mathrm{C}_{\max }$. This ISCV should not be the inclusion of outlier and the same to be justified by the calculated intra-subject variability is a reliable estimate by the applicants (Table 1).

\section{In Vitro dissolution tests}

As per the EMA \& GCC requirements, bioequivalence used same batch/lot No. of test and reference products multi-medium dissolution (generally $\mathrm{pH} 1.2, \mathrm{pH} 4.5 \& \mathrm{pH} 6.8$ ) and release medium reports should be reported. USFDA recommends that the appropriate USP method should be submitted. If there is no USP method available, we recommend that the FDA method for the reference listed drug be used. European Medicines Agency, London, 20 January 2010, (Doc. Ref.: CPMP/EWP/QWP/1401/98 Rev. 1/ Corr), Committee for Medicinal Products for Human Use, Guideline on the investigation of bioequivalence. Guidance for Industry Bioequivalence Studies with Pharmacokinetic Endpoints for Drugs Submitted Under an ANDA, Draft Guidance, U.S. Department of Health and Human Services Food and Drug Administration Center for Drug Evaluation and Research (CDER), Biopharmaceutics, December 2013. Executive Board of the Health Ministers' Council for GCC States, the GCC Guidelines for Bioequivalence, Version 2.4, MAR 2016 [4].

\section{BCS-based Biowaiver}

In U.S. biowaivers are applicable based on Biopharmaceutical classification system (BCS) for BCS class 1 and class 3 IR solid oral

Table 1: This ISCV should not be the inclusion of outlier and the same to be justified by the calculated intra-subject variability is a reliable estimate by the applicants.

\begin{tabular}{ccc}
\hline Within-subject CV (\%) & Lower CI & Upper CI \\
\hline 30 & 80.00 & 125.00 \\
\hline 35 & 77.23 & 129.48 \\
\hline 40 & 74.62 & 134.02 \\
\hline 45 & 72.15 & 138.59 \\
\hline 50 & 69.84 & 143.19 \\
\hline
\end{tabular}


dosage forms. As per the FDA guidance, Biowaiver is acceptable for BCS class 1 drug products, if the below points are demonstrated:

- Drug substance should be highly soluble

- Drug substance should be highly permeable

- Drug products are rapidly dissolving, and

- The product doesn't have any excipients that will affect the rate or extent of absorption of the drug

Biowaiver is acceptable for BCS class 3 drug products, if the below points are demonstrated:

- Drug substance should be highly soluble

- Drug products should be very rapidly dissolving and

- The test product formulation is qualitatively the same and quantitatively very similar

BCS biowaiver applicability for IR products in EMA for both BCS class 1 and BCS 3 products in consideration of the below requirements.

For BCS class 1 drug products, the below mentioned points should be demonstrated:

- Drug substance should have high solubility and complete absorption and

- Drug product should have very rapid dissolving property (more than $85 \%$ within $15 \mathrm{~min}$ ) or rapidly dissolving (more than $85 \%$ within $30 \mathrm{~min}$ ) considering specific requirements

- Excipients used in the formulation that might affect bioavailability are qualitatively and quantitatively the same and usually similar amount of same excipients are preferred.

For BCS class 3 drug products, the below mentioned points should be demonstrated:

- Drug substance should have highly soluble and limited absorption and

- Drug product should have very rapidly dissolving property (>85\% within $15 \mathrm{~min}$ ) considering specific requirements

- Excipients used in the formulation that might affect bioavailability are qualitatively and quantitatively the same and other excipients are qualitatively the same and quantitatively very similar.

In GCC, BCS biowaiver applicability is possible for BCS class 1 immediate release products in consideration of the below mentioned points to be proved.

- Drug substance should highly soluble and completely absorbed

- Drug product should have very rapid solubility or rapid solubility in in-vitro dissolutions for both test and reference products and

- If the using excipients have any impact in bioavailability and then it should be similar in the aspects of qualitative and quantitative. Generally, similar quantity is advisable for the same excipients.

In addition to that, Saudi FDA "Guidelines for Biowaiver Based on Biopharmaceutics Classification System (BCS) For ImmediateRelease (IR) Solid Oral Dosage Forms" states that, "Biowaiver may be applicable when the active substance(s) in test and reference products are identical and belong to BCS- Class I on Biowaiver monographs published by International Pharmaceutical Federation (FIP) or in FDA website under "Bioequivalence recommendations for specific product". In the case of biowaiver consideration these sources are taken in to account". Saudi FDA guidelines has given the clarity on the selection of the molecules for biowaiver applicability. USFDA and EMA both they are considering the all BCS class I molecules for the biowaiver applicability based on the fulfillment of their guidance requirements. Moreover, both USFDA \& EMA they are considering the biowaiver applicability for BCS class III molecules. Hence, this biowaiver requirements harmonization in these region will be helpful to avoid the unnecessary additional tests in the human for the highly soluble molecules.

European Medicines Agency, London, 20 January 2010, (Doc. Ref.: CPMP/EWP/QWP/1401/98 Rev. 1/ Corr), Committee for Medicinal Products for Human Use, Guideline on the investigation of bioequivalence.

Guidance for Industry BioequivalenceStudies with Pharmacokinetic Endpoints for Drugs Submitted Under an ANDA, Draft Guidance, U.S. Department of Health and Human Services Food and Drug Administration Center for Drug Evaluation and Research (CDER), Biopharmaceutics, December 2013.

Executive Board of the Health Ministers' Council for GCC States, the GCC Guidelines for Bioequivalence, Version 2.4, MAR 2016.

Saudi Food and Drug Authority, Guidelines for Biowaiver Based on Biopharmaceutics Classification System (BCS) For ImmediateRelease (IR) Solid Oral Dosage Forms, Version 1.1 (Final \& updated) [5].

\section{CONCLUSION}

I hope, this article has provided a basic prompt overview for comparing important parameters of the bioequivalence regulatory requirements of the USFDA, EMA and GCC. In addition, it describes the important differences and similarities in $\mathrm{BE}$ approaches for the regulatory agencies of EMA, GCC and USFDA. Moreover, it briefly compares and given the prompt overview of the bioequivalence study requirements in these region and in comparison with the USFDA and EMA (Table 2). Since the GCC guidelines adherence to the EMA and hence no difference between both regulatory requirements. EMA and GCC are having both similarities and differences in comparison with USFDA guidance requirements. At present EMA regulatory also initiated the product specific guidance as like USFDA. This approach helps to understand the clear way forward to the marketing authorization holders towards filing in the EMA. However, as like EMA bioequivalence guidelines adherence by GCC regulatory, currently need to incorporate important advances in the bioequivalence, including product specific guidance and BCS based biowaivers for class III molecules would be the important step towards global harmonization of bioequivalence and also important for the regional generic drug approval process. 
Table 2: Prompt overview on BA BE studies requirements in the GCC, FDA and EMA regulatory regions

Country Regulatory

Gulf Cooperation Council (GCC) (Executive

Board of the Health Ministers Council for GCC United States Food
States)

Saudi Arabia, Kuwait, The United Arab

Countries

Emirates, Qatar, Bahrain, and Oman

Compared, a randomized, two-period, twosequence single dose crossover design $n$

Design of the study

Study design for Long half life drugs

Parallel design for substances with very long half-life

Study design for Highly Replicate designs for substances with highly variable drugs variable pharmacokinetic characteristics

Sample Size

24 subjects for pivotal bioequivalence study

Non-replicate crossover study design

If any issues in crossover study, study

design with parallel approach can be used

With mixed scaling, the reference- Replicate designs for scaled form of the criterion should be substances with highly variable used

Pilot: Small number of subjects Pivotal: Not specified

Selection of Reference Original brand-name (i.e. manufactured in the As per the orange book (RLD) drug

country of origin of the original brand name).

Replacement of subjects

on withdrawal or dropout

No replacements for dropouts

requirements.

Not specified

Both sex

Gender of the Subject Subjects could belong to either sex

The risk to women of childbearing potential should be considered

Study specific requirements.

Female Subjects

Subjects should be 18-55 years of age

18 years of age or older

Age criteria

Subjects should be 18-55 years of age

BMI

History of Smoking

Criteria for Selection

History of Alcohol and

Drug Abuse Criteria for Selection

Body Mass Index between

18.5 and $30 \mathrm{~kg} / \mathrm{Sq} . \mathrm{m}$.

Not specified

Subjects should preferably be non-smokers

Not specified

Subjects should preferably be without a history Not specified of alcohol or drug abuse.

\section{Restrictions (Smoking/}

Alc ohol and Drug Not specified

Abuse) administration of the products, unless otherwise Overnight fast of at least 10 hours justified.
Abstain from alcohol for 24 hours before each study period and until after the last sample from each period is collected pharmacokinetic Characteristics 12 subjects for bioequivalence study

Europe

Compared, a randomized, twoperiod, two-sequence single dose crossover design

Parallel design for substances with very long half-life

Europe marketed product

Not specified

Subjects could belong to either sex

The risk to women of Childbearing potential should be considered.

Subjects should be 18 years of age or older

Body Mass Index between

18.5 and $30 \mathrm{~kg} / \mathrm{m} 2$.

Subjects should preferably be non-smokers

Subjects should preferably be without a history of alcohol or drug abuse.

\section{Not specified}

Subjects should fast for at least 8 hours prior to administration of the products, unless otherwise justified.

A high-fat (approximately 50 percent A high-fat (approximately 50

A high-fat (approximately 50 percent of total of total caloric content of the meal) percent of total caloric content and high-calorie (approximately 800 to of the meal) and high-calorie caloric content of the meal) and high-calorie

Food Specification for (approximately 800 to $1000 \mathrm{kcal}$ ) meal. This Fed Studies test meal should derive approximately 150,250 , and 500-600 kcal from protein, carbohydrate, and fat, respectively.

000 calories) meal is recommended as (approximately 800 to 1000 a test meal for food-effect BA and fed kcal) meal. This test meal BE studies. This test meal should derive should derive approximately approximately 150, 250, and 500-600 150, 250, and 500-600 kcal from calories from protein, carbohydrate, protein, carbohydrate, and fat, and fat, respectively.

A sufficient number of samples to adequately

Number of Samples describe the plasma concentration-time profile 12 to 18 samples should be collected. respectively.

A sufficient number of samples to adequately describe the plasma concentration-time profile should be collected.

It is recommended that water is allowed as Subjects be allowed water as desired It is recommended that water is

Water Restriction desired except for one hour
after drug administration

Pre-dose concentration is greater than 5 percent

Subjects with Pre-dose plasma conc. of the $\mathrm{C}_{\max }$ value for the subject in that period, Recommend that if the predose value is the statistical analysis should be performed $>$ than 5 percent of $\mathrm{C}_{\max }$, the subject be with the data from that subject for that period dropped from all BE study evaluations. excluded. allowed as desired except for one hour before and one hour after drug administration

Pre-dose concentration is greater than 5 percent of the $\mathrm{C}_{\text {max }}$ value for the subject in that period, the statistical analysis should be performed with the data from that subject for that period excluded. 
Fluid (water) intake at Dosing

Posture

Plasma storage
The test and reference products should be The test or reference products can be administered with a standardized volume of administered with about 8 ounces ( 240 fluid (at least $150 \mathrm{ml}$ ).

The test and reference products should be administered with a standardised volume of fluid (at least $150 \mathrm{ml}$ ).

Posture and physical activity may need to be Not specified
standardized. standardized.

Posture and physical activity may need to be standardised.

Not specified

An adequate washout period (e.g.,

Wash-out period Not specified Normally at least 5 elimination longest half- more than 5 half lives of the moieties lives are necessary to achieve this.

to be measured) would separate each treatment.

Pharmacokinet ic AUC0-t, AUC0- $\infty, \mathrm{C}_{\text {max }}$, AUC0-t, AUC0- $\infty, \mathrm{C}_{\max }$, parameters $\mathrm{T}_{\max }, \lambda \mathrm{z}$, and $\mathrm{t} 1 / 2$ $\mathrm{T}_{\max }, \lambda \mathrm{z}$, and $\mathrm{t} 1 / 2$ Normally at least 5 elimination half-lives are necessary to achieve this.

AUC0-t, AUC0- $\infty, \mathrm{C}_{\max }$, $\mathrm{T}_{\max }, \lambda z$, and $\mathrm{t} 1 / 2$

Pharmacokinet ic parameters for steady state AUC $(0-\tau), \quad \mathrm{C}_{\max s,}$ and $\mathrm{t}_{\text {mos }}$ should be $\mathrm{C}_{\min }$, Cav, degree of fluctuation $\left[\left(\mathrm{C}_{\mathrm{m}}\right.\right.$ AUC $(0-\tau), \mathrm{C}_{\text {max,ss }}, \quad$ and $t_{\text {max,ss }}$ should be $\mathrm{C}_{\text {min }}$ ) $\left./ \mathrm{Cav}\right]$, and swing [(Cmax-Cmin)
determined. Cmin]

Pharmacokinet ic parameters for when using urinary data

Ae(0-t) and, if applicable, $R_{\text {max }}$ should be Study specific guidance. determined.

$\operatorname{AUC}(0-\tau), \quad \mathrm{C}_{\max _{\mathrm{xs}},}$ and $\mathrm{t}_{\mathrm{max} s \mathrm{ss}}$ should be determined.

$\mathrm{Ae}(0-\mathrm{t})$ and, if applicable, $\mathrm{R}_{\mathrm{max}}$ should be determined.
Statistical evaluation

Bioequivalence is based upon $90 \%$ confidence intervals for the ratio of the population geometric means (test/reference) for the parameters under consideration.
USFDA recommends that the statistical information be provided for AUC0-t,

$\mathrm{AUC} 0-\infty$, and $\mathrm{C}_{\max }$ :

- Geometric mean

- Arithmetic mean

- Ratio of means

- Confidence intervals

$90 \%$ confidence interval of $80.00-90 \%$ confidence interval of $80.00-$ $125.00 \%$ $125.00 \%$

AUC ratio, ${ }_{\text {Cmax }}$ etc.

$90 \%$ confidence interval of $80.00-125.00 \%$

AUC \& $\mathrm{C}_{\max }$ should be tightened

USFDA guidance recommends the $\mathrm{BE}$ to 90.00 limit of 80 to 125 percent

AUC ratio, $C_{\max }$ for $\mathrm{AUC} \& \mathrm{C}_{\max }$ should be tightened to 90.00 -
Bioequivalence is based upon ratio of the population geometric means (test/reference) for the parameters under consideration.
Narrow therapeutic range $111.11 \%$ drugs.

Acceptance criteria for AUC ratio, $\mathrm{C}_{\text {max }}$ for Highly variable drugs

Limitation for Blood Withdrawal Quantity

Base line correction

pre-dose concentrations or

pre-dose concentrations or
subtraction of the individual endogenous predose AUC, is preferred

A wider acceptance range (i.e. $75-133 \%$ ) for $\mathrm{C}_{\max }$ can be used

Not specified

Subtraction of the mean of individual Subtract these levels from the total Subtraction of the mean of concentrations measured from each individual endogenous pre- dose subject

The reference-scaled average $\mathrm{BE}$ approach adjusts the BE limits of 0.8 to 1.25 on the geometric mean ratio
Not specified
Not specified

A wider acceptance range (i.e. $75-133 \%$ ) for $\mathrm{C}_{\text {max }}$ can be used concentrations or subtraction of the individual endogenous pre- dose AUC, is preferred administered Part

Active drug ingredient or its active

Measurement of metabolite

Parent compound. However, it is acceptable for the main active metabolite

(parent in the administered dosage form active metabolites

Subject data deleted from statistical analysis if vomiting occurs at or before

Data deletion due to vomiting

Exclude the results from a subject in a particular 2 times median Tmax. For, modifiedperiod are events such as vomiting and diarrhoea release products, emesis any time during the labeled dosing interval can be deleted

\section{Difference in the drug}

proportion between Test Not more than 5\% and Reference

\section{Period for retention of
drugs Not specified}

Quantity of drugs to be Not specified
retained

Period for retention of documents
Not more than 5\%

Not more than $5 \%$
Parent compound. However, it is acceptable for the main active metabolite

Exclude the results from a subject in a particular period are events such as vomiting and diarrhoea 


\section{GLOSSARY OF TERMS}

A generic drug is the same as a brand name drug in dosage, safety, strength, how it is taken, quality, performance, and intended use. Before approving a generic drug product, FDA requires many rigorous tests and procedures to assure that the generic drug can be substituted for the brand name drug. The FDA bases evaluations of Generic Drug1 substitutability, or "therapeutic equivalence," of generic drugs on scientific evaluations. By law, a generic drug product must contain the identical amounts of the same active ingredient(s) as the brand name product. Drug products evaluated as "therapeutically equivalent" can be expected to have equal effect and no difference when substituted for the brand name product.

A Reference Listed Drug (RLD) is an approved drug product to which new generic versions are compared to show that they are bioequivalent. A drug company seeking approval to market a generic equivalent must refer to the Reference Listed

RLD (Reference Drug in its Abbreviated New Drug Application (ANDA). By designating a single reference listed drug as the standard to which all generic versions must be shown to be bioequivalent, FDA hopes to avoid possible significant variations among generic drugs and their brand name counterpart.

The rate and extent to which the active ingredient or active moiety is absorbed from a drug product and becomes available at the site of action. For drug products that are not intended to be

Bioavailability absorbed into the bloodstream, bioavailability may be assessed by measurements intended to reflect the rate and extent to which the active ingredient or active moiety becomes available at the site of action.

The absence of a significant difference in the rate and extent to which the active

ingredient or active moiety in pharmaceutical

Bioequivalence equivalents or pharmaceutical

alternatives becomes available at the site of drug action when administered at the

same molar dose under similar conditions in an appropriately designed study.

\section{Gulf}

Cooperation Council
Gulf Cooperation Council (GCC) (Executive Board of the Health Ministers Council for GCC States). Saudi Arabia, Kuwait, The United Arab Emirates, Qatar, Bahrain, and Oman
Nonreplicated The standard two-formulation, two-period, twoDesigns sequence crossover design

Replicated For this design, the same lots of the $\mathrm{T}$ and $\mathrm{R}$ Crossover formulations should be used for the replicated administration. Each period should be separated by an adequate washout period.

US Food and Drug Administration, Drugs@FDA Glossary of Terms [6].

Guidance for Industry, Bioequivalence Studies with Pharmacokinetic Endpoints for Drugs Submitted Under an ANDA, Draft Guidance, U.S. Department of Health and Human Services Food and Drug Administration, Center for Drug Evaluation and Research (CDER), December 2013.

Guidance for Industry Bioavailability and Bioequivalence Studies for Orally Administered Drug Products - General Considerations, U.S. Department of Health and Human Services Food and Drug Administration, Center for Drug Evaluation and Research (CDER) [7].

\section{REFERENCES}

1. Guidance for Industry, Handling and Retention of BA and BE Testing Samples, U.S. Department of Health and Human Services, Food and Drug Administration, Center for Drug Evaluation and Research (CDER), May 2004.

2. European Medicines Agency, London, 20 January 2010, (Doc. Ref.: CPMP/EWP/QWP/1401/98 Rev. 1/ Corr), Committee for Medicinal Products for Human Use, Guideline on the investigation of bioequivalence.

3. Executive Board of the Health Ministers' Council for GCC States, the GCC Guidelines for Bioequivalence, Version 2.4, MARCH 2016.

4. Guidance for Industry Bioequivalence Studies with Pharmacokinetic Endpoints for Drugs Submitted Under an ANDA, Draft Guidance, U.S. Department of Health and Human Services Food and Drug Administration Center for Drug Evaluation and Research (CDER), Biopharmaceutics.

5. Saudi Food and Drug Authority, Guidelines for Biowaiver Based on Biopharmaceutics Classification System (BCS) For ImmediateRelease (IR) Solid Oral Dosage Forms, Version 1.1 (Final \& updated), 15/07/2013.

6. US Food and Drug Administration, Drugs@FDA Glossary of Terms.

7. Guidance for Industry Bioavailability and Bioequivalence Studies for Orally Administered Drug Products - General Considerations, U.S. Department of Health and Human Services Food and Drug Administration Center for Drug Evaluation and Research (CDER), BP, Revision 1, March 2003. 